\title{
Design and Implementation Challenges of Microelectrode Arrays: A Review
}

\section{Bahareh Ghane-Motlagh, Mohamad Sawan}

Polystim Neurotechnologies Laboratory, Department of Electrical Engineering, Polytechnique Montreal, Montreal, Canada. Email: bahareh.ghane-motlagh@polymtl.ca

Received June $5^{\text {th }}, 2013$; revised July $4^{\text {th }}, 2013$; accepted July $16^{\text {th }}, 2013$

Copyright (C) 2013 Bahareh Ghane-Motlagh, Mohamad Sawan. This is an open access article distributed under the Creative Commons Attribution License, which permits unrestricted use, distribution, and reproduction in any medium, provided the original work is properly cited.

\begin{abstract}
The emerging field of neuroprosthetics is focused on design and implementation of neural prostheses to restore some of the lost neural functions. Remarkable progress has been reported at most bioelectronic levels - particularly the various brain-machine interfaces (BMIs) - but the electrode-tissue contacts (ETCs) remain one of the major obstacles. The success of these BMIs relies on electrodes which are in contact with the neural tissue. Biological response to chronic implantation of Microelectrode arrays (MEAs) is an essential factor in determining a successful electrode design. By altering the material compositions and geometries of the arrays, fabrication techniques of MEAs insuring these ETCs try to obtain consistent recording signals from small groups of neurons without losing microstimulation capabilities, while maintaining low-impedance pathways for charge injection, high-charge transfer, and high-spatial resolution in recent years. So far, none of these attempts have led to a major breakthrough. Clearly, much work still needs to be done to accept a standard model of MEAs for clinical purposes. In this paper, we review different microfabrication techniques of MEAs with their advantages and drawbacks, and comment on various coating materials to enhance electrode performance. Then, we propose high-density, three-dimensional (3D), silicon-based MEAs using micromachining methods. The geometries that will be used include arrays of penetrating variable-height probes.
\end{abstract}

Keywords: Microelectrode Arrays; Electrodes-Tissues Contacts; Microelectrode Impedance; Neural Prosthesis

\section{Introduction}

Nervous system disorders reduce the quality of life of afflicted persons - and are the most difficult to treat of all parts of the human body. Most of these impairments result from lost localized sensory or motor dysfunction. The evolving field of neuroprosthetics focuses on design and implementation of neural prostheses such as visual and cochlear implants to restore lost neural function by recording signals from active neurons and subsequent selective electrical stimulation of a population of neurons [1]. Images or sounds taken from cameras or microphones are coded to electrical impulses to stimulate retinal tissues for the blind or auditory nerves for the deaf. In another type of neuroprosthesis, electrical signals are sent to a targeted portion of a patient's brain suffering from epilepsy, Parkinson's disease, or depression to decrease the effects of the condition.

Motor neuroprostheses control external devices such as prosthetic limbs with signals from the central or peripheral nervous systems. The ones that interface with the central nervous system are called brain-machine interfaces (BMIs) [2]. The most critical component in this function restoration is a microelectrode array (MEA) implanted in neural tissue, which acts as an electrode-neural tissues interface. In addition to injecting a charge during stimulation, the ultimate role of MEAs is to provide precise measurements of neural activity [3].

The field of implantable BMI based on MEAs is an emerging research activity. Remarkable progress has been reported at most bioelectronic levels, but the electrode-tissue contacts (ETCs) remain one of the major obstacles. Contacts achieved using MEAs do not comply with the remaining parts of these BMIs due to the biological response to chronic implantation and to the electronic properties of MEAs. The success of these BMIs relies on electrodes which are in contact with the neural tissue. However, design and fabrication of a convenient interface with selectivity, good electric characteristics, sensitivity, biocompatibility, and long-term chemical and recording stability remain a tough challenge [4]. For 
electrical stimulation and recording, we need electrodes with high selectivity, low impedance, high-density, and multi-dimensional geometry. Electrodes with high selectivity and low impedance are preferred to stimulate specific nerve cells and to achieve a high signal-to-noise ratio (SNR). For higher selectivity, however, a smaller electrode size is required; this results in higher impedance and a lower SNR [5]. To address this issues, we should consider the entire combination of main aspects - design, materials, and fabrication techniques.

To date, implantable MEAs have been fabricated by three common techniques: microwire, micromachined, and flexible arrays [6]. Microwires, made from tungsten or stainless steel, were the first implantable electrodes to record chronically from the brain. This technique is used to focus on the individual neuron [7]. One particular advantage of microwires is that they can be applied to access deep brain structures. By using the arrays of microwires, simultaneous recording at the level of neuronal populations is possible. One drawback of these electrodes is that the accurate location of the electrode tips relative to each other is not controllable because of the wire bending during implantation. Micromachined electrode arrays can be silicon or metal based. The silicon-based electrodes include two specific models. In the first model, microelectrode sites are patterned on each shank of silicon micro-machined substrate. This technique provides a higher density of sensors, which reduces tissue displacement compared to microwires and contains active electronics integrated into the arrays [8]. The second model, however, includes sharpened silicon needles electrically isolated from each other. The tips are coated with platinum or iridium oxide; the rest are insulated with polyamide. These electrodes are designed to be implanted in the cerebral cortex or peripheral nerves [9].

Another type of micromachined electrode is a metal-based array created by an electrical discharge machining $(\mathrm{EDM})$ technique associated with electrochemical steps. In this method, electrodes are made by micromachining a piece of stainless steel. After forming the electrodes, platinum is deposited on the surface of the tips of the electrodes which are electrically insulated from each other by applying epoxy between them $[10,11]$.

A flexible multi-electrode array is another type of electrode. In this technique, the metal-based electrodes are covered by two layers of polyimide, parylene, or benzocycobutene without a silicon structure. Flexible arrays provide an advantage over rigid electrode arrays because of the closer mechanical match with brain tissue. The mechanical mismatch between the rigid probes and soft brain tissue can cause inflammation in the places of implantation [12]. The flexible nature of these electrodes, however, involves some difficulties during insertion. Polymer-based implantable electrodes are a new ap- proach to modify traditional electrode recording sites to improve long-term performance [5].

In recent years, MEA techniques have been developed to have a long-term and stable interface with the brain. Different research projects are conducted to obtain consistent recording signals from small groups of neurons without losing microstimulation capabilities, while maintaining low-impedance pathways for charge injection, high-charge transfer, and high-spatial resolution by altering the material compositions and geometries of the arrays [13]. So far, none of these attempts have led to a major breakthrough. One of the main challenges in fabricating MEAs is finding appropriate coating materials which will be biocompatible and improve neural recordings. Conducting polymers and carbon nanotubes (CNTs) have attracted much interest as suitable materials for coating the electrodes. They ameliorate neural recordings by decreasing electrode impedance and increasing charge transfer but they cannot eliminate glial encapsulation [4]. The molecular/cellular biology approach attempts to minimize the immune response to implanted electrodes by using bioactive molecules. Such an approach is focused on coating electrodes with bioactive molecules [14]. To achieve these goals, we propose 3D, high-density, silicon-based MEAs using micromachining techniques. The geometries that will be used include arrays of penetrating variable-height probes. These arrays can selectively communicate with large numbers of individual neurons. To this purpose, the tips of sharpened silicon needles will be coated with conducting polymers using electrochemical techniques to enhance both recording and electrical stimulation by decreasing the electrode impedance and increasing charge transfer. In this review, we describe the technology and design of different types of microelectrode arrays with their advantages and drawbacks in Section 2. In Section 3 we focus on about electrode-tissue interface, tissue reaction to implantable electrodes which is referred to the biocompatibility of the implant, and some of the potential strategies to improve the biocompatibility of implanted cortical electrodes. Then, we propose our MEAs using micromachining method.

\section{Types of Electrodes}

The design and implementation of appropriate implantable neural interfaces for electrical recording and efforts to understand the mechanism of the nervous system at the cellular level was begun in the last century; however, research on using electrical stimulation to restore movement of limbs was started more than two centuries ago $[15,16]$. Neural interfaces are used to understand physiological processes at the cellular level and to restore sensory or motor function in the nervous system. Significant progress has been achieved in the first part but there has 
been a lack of technological advances at restoration nervous system dysfunction.

\subsection{Microwire Arrays}

The first implantable electrodes as an electronic interface were made from sharpened wire metal in the 1950s. Their narrow structures let them be placed very close to single neurons in vivo, causing minimal damage in tissue. They are insulated, except for the tips, to record the highly localized extracellular potentials from the neurons closest to the tips and also to inject the localized stimulation current to excite the nearest neurons [17]. Metal electrodes are fabricated from different types of materials such as stainless steel, tungsten, platinum, iridium, or gold. Strumwasser used $80-\mu \mathrm{m}$ diameter stainless steel wires to record discharges from single neurons of unanesthetized animals for a week or more [7]. Electrolytically sharpened tungsten wire electrodes with platinised tips were used for extracellular single recording by Robinson in 1968. The platinising process - a spongy deposit of platinum black on the tips-enhances the effective surface area and causes metal microelectrodes to achieve their priority over glass pipettes [18]. Since stainless steel is fragile near the tips, tungsten was replaced due to the stiffness and rugged structure, and to provide very stable recordings; however, tungsten is very noisy at low frequencies. Signals of the mammalian nervous system compared to other biological signals are fast and, by using a high-pass filter to remove noise, slow signals can be lost; thus, tungsten is not a good choice for recording these kinds of signals [19]. Metal electrodes have less SNR because of lower impedance for the frequency range of spike signals. A platinum electrode plated with platinum black gives stable recordings, high SNR, and creates a porous low-impedance structure, but it is mechanically fragile [20]. Iridium metal wire is extremely stiff, highly resistance to corrosion, and its surface is electrochemically activated, which causes it to increase the maximum charge density [17].

Microwires remain in use today and fabrication methods have not changed basically. They give long-lasting individual neurons recording, sometimes more than one year, so they allow neuroscientists to focus on individual neurons. Using arrays of microwires allows multi-neural recordings from neuronal populations [21]. Therefore, simultaneous recordings from a large number of neurons will be provided. Figure 1 shows a typical microwire arrays fabricated by Plexon.

In all the above-cited cases, the final contact between electrodes and brain tissue is a metal. Recently, CNTs and polymers have been used to coat the tips of the metal wire electrodes. In 2008, a group of researchers in Texas coated the tips of conventional tungsten and stainless steel wire electrodes with a combination of CNTs and

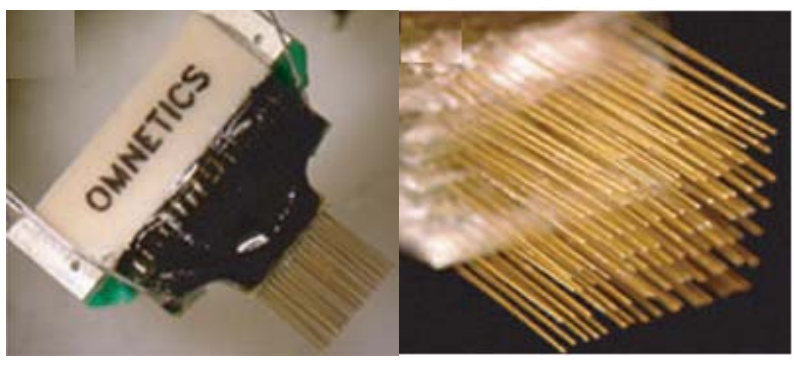

(a)

(b)

Figure 1. Implantable plexon microwires: (a) Microwire arrays attached to the high-density connector; (b) High-density microwire arrays [18].

polymer for the first time. They deposited CNTs on indium-tin oxide MEAs [22]. Measurements showed that the impedance decreased from $940 \mathrm{k} \Omega$ to $38 \mathrm{k} \Omega$ at a frequency of $1 \mathrm{kHz}$, and showed $\sim 40$-fold increase in charge transfer before and after coating (Figure 2).

CNT coatings can be applied to different materials and geometries. In Figure 3 sharpened tungsten and stainless steel wire electrodes were coated with CNTs.

The electrical characterization of modified tungsten and stainless steel electrodes by electrochemical deposition of a covalent attachment scheme $(\mathrm{CNT} / \mathrm{CoV})$, and $\mathrm{CNT} /$ polypyrrole (CNT/Ppy) composite are shown in Table 1. Multi-walled carbon nanotubes (MWCNTs) add to Ppy throught two different methods: layering and codeposition. The advantage of this combination is that the MWCNTs are restricted within the polymer matrix, preventing MWCNTs accumulation or mobilisation surrended by cellular tissues [23]. CNT-coated electrodes not only provide an appropriate substrate for neural growth and function for at least three months but also improve both the recording and stimulating characteristics of neural electrodes [4].

One of the advantages of metal electrodes is access to deep brain structures to treat disorders such as epilepsy, Parkinson's disease, and paralysis. By using arrays of microwires, simultaneous recording at the level of neural populations is possible [21]. One particular disadvantage of microwires is bending of the wires during implantation, so the accurate location of the electrode tips relative to each other is not controllable [6]. There is another issue with metal electrodes - they are not always compatible with silicon-based integrated circuits.

\subsection{Micromachined Arrays}

\subsubsection{Silicon-Based Multi-Shaft Array}

With the invention of the lithography technique in 1959, which provided small size and accurate dimensional control for integrated circuit fabrication and use of this process for fabricating extracellular MEAs, a new way for simultaneously recording from many neurons, or 


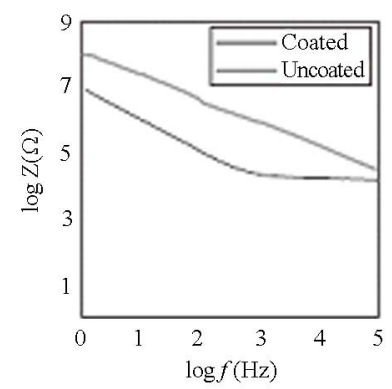

(a)

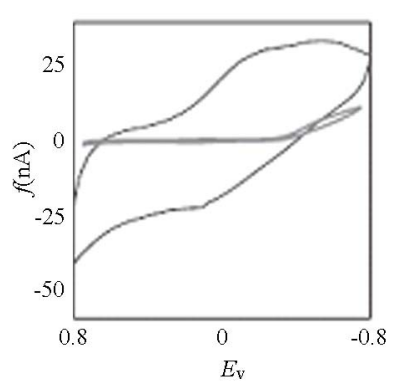

(b)
Figure 2. Characteristics of CNT-coated MEAs: (a) An impedance spectroscopy scan; (b) A cyclic voltammetry scan [4].

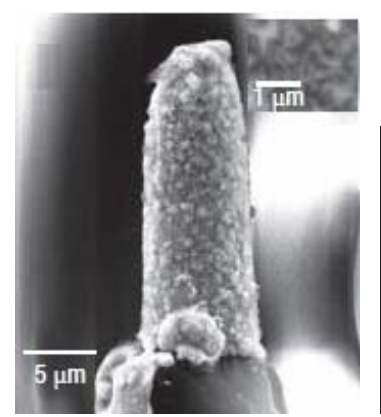

(a)

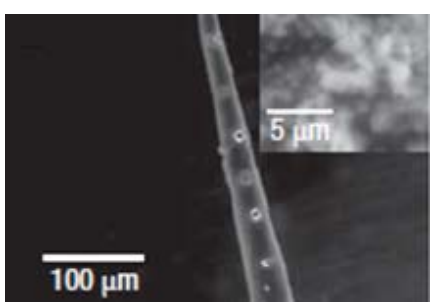

(b)
Figure 3. Metal electrodes coated with CNTs: (a) CNTs covalently attached to the sharp tungsten electrode, (b) Parylene insulation exposed stainless steel electrode removed by UV laser, polymerized with a mixture of CNTs/ Polypyrrole composite [4].

stimulate them, was provided $[24,25]$. Use of the lithographic technique and silicon etching technology to create high-density electrode arrays for single-unit recording began at Stanford University in 1966. By the late 1960s, these new technologies were used by Brindley to fabricate visual prostheses and they were developed as a cortical prosthesis for the blind at the University of Utah $[26,27]$. Silicon-based electrodes intended for both long-term monitoring of neural activity and injection of stimuli into neural tissues at the cellular level are compatible with CMOS technology, which uses planar photolithographic techniques on silicon wafers. Despite the problem that microwire tips may move apart during implantation, the spatial relation between electrodes in microfabricated arrays is determined during the microfabrication process and remains fixed. One of the first silicon-based MEAs was made at Michigan University during the 1970s. In this design, electrode sites are placed on each shank. This kind of electrode can easily merge with on-chip circuitry, signal processing, and wireless interfaces. Figures $\mathbf{4}$ and $\mathbf{5}$ show the basic structure of a micro-machined microelectrode probe for recording or stimulation in the central nervous system and a four-pe-
Table 1. Electrical properties of nanotube-modified electodes [4].

\begin{tabular}{lcc}
\hline & $\begin{array}{c}\text { Normalized impedance } \\
\left(\Omega \cdot \mathrm{cm}^{-2}\right)\end{array}$ & $\begin{array}{c}\text { Normalized capacitance } \\
\left(\mathrm{mF} \cdot \mathrm{cm}^{-2}\right)\end{array}$ \\
\hline $\mathrm{CNT} / \mathrm{CoV}$ & 0.075 & 38 \\
$\mathrm{CNT} / \mathrm{Ppy}$ & 0.77 & 755 \\
\hline
\end{tabular}

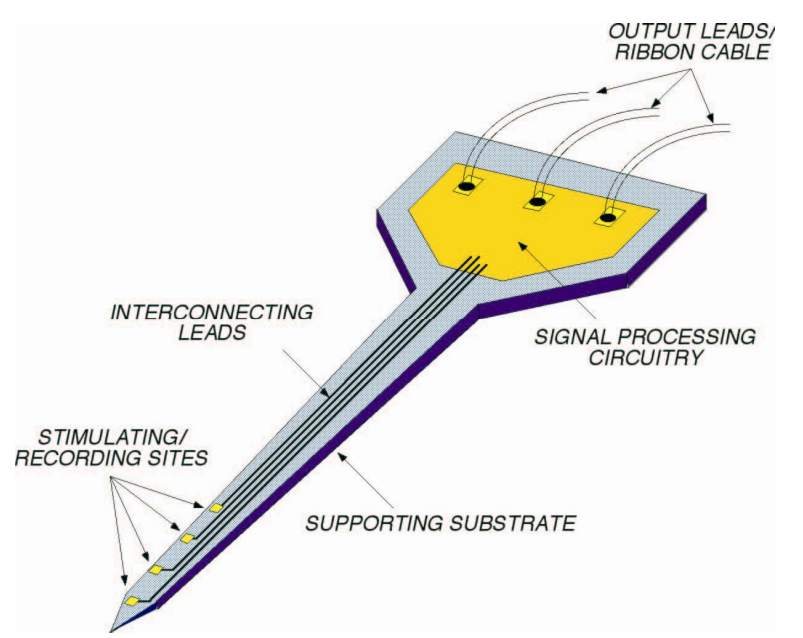

Figure 4. Typical Michigan probe: Basic structure of a micromachined microelectrode probe [29].

netrating-shank 16-channel probe with four uniformly recording sites, respectively $[28,29]$. The silicon substrate shank is $15 \mu \mathrm{m}$ thick, $3 \mathrm{~mm}$ long, and $90 \mu \mathrm{m}$ wide at the base, narrowing to $20 \mu \mathrm{m}$ at the tip. The surface area of the recording sites is between 100 to $400 \mu \mathrm{m}^{2}$. The high-density, 3D electrode arrays are inserted in the tissue to record electrical activity or inject electrical signals to stimulate neurons. The substrate of the probe (shank) should be biocompatible, strong, and small enough to penetrate to the pia arachnoid membrane over the brain. Silicon is strong enough and allows integrated electronics to be formed directly in the probe substrate [30].

The recording sites are typically made of iridium (Ir) or gold. These recording sites can be modified by coating with conductive polymers such as Ppy or poly (3, or 4ethylenedioxythiophene-PEDOT) to improve long-term performance and decrease recording site impedance. Experimental and theoretical characterization of these microelectrodes modified with conducting polymer nanotubes showed a significant impedance decrease and a charge injection capacity increase by two and three orders of magnitude, respectively. The impedance spectroscopy results of PEDOT nanotube-coated electrode sites with applied charge inject density of $1.4 \mathrm{C} / \mathrm{cm}^{2}$ are shown in Figure 6.

The charge capacity of bare gold, Ppy, and PEDOT electrodes are shown in Table 2 [31].

According to the CNT-related results, which included 


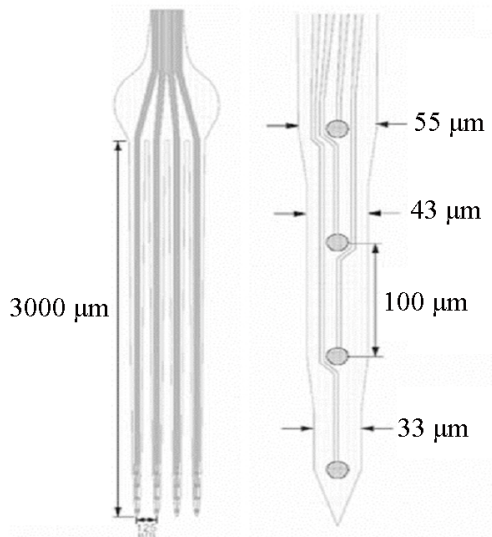

Figure 5. Typical Michigan probe: General schematic of the four-shank electrodes forming16-channel probe [28].
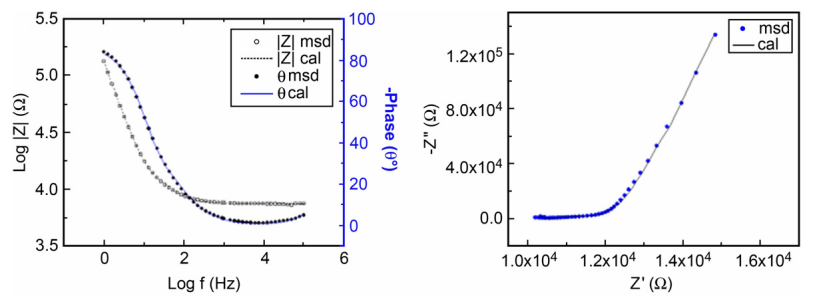

Figure 6. Measured and calculated data of PEDOT nanotubes impedance with applied charge density of $1.4 \mathrm{C} / \mathrm{cm}^{2}$ : (a) Bode plot; (b) Nyquist plot [31].

decreasing the impedance and noise, the recording sites were modified by CNTs coating [32]. The results showed that CNTs incorporation by increasing the effective surface area could decrease site-tissue impedance [33].

Novel, multi-sided parylene-based MEAs with electrode sites in the front-side, back-side, and on the edge of the device was fabricated for neural recording. This method can increase the function of each shank [34].

One of the advantages of these microelectrodes is providing a high density of sensors with predetermined locations and high spatial resolution. There are some issues with this kind of electrode array: the shank-which includes recording sites - causes more tissue displacement and may damage some neurons during insertion; also, dura mater should be partially removed for inserting the electrode arrays (Figure 7). The voluminous electronic structure, which assembles the electrode shanks, causes more damage in the brain tissue during insertion to the cortex.

\subsubsection{Silicon-Based Multi-Needle Array}

Another type of silicon-based microelectrodes is Utah arrays which consist of conductive, sharpened silicon needles electrically isolated from each other. The maximum length of the needles is $1.5 \mathrm{~mm}$. The tips of the needles are coated with platinum or iridium oxide; the rest are insulated with biocompatible polymers such as polyimide or parylene-C. The architecture of these elec-
Table 2. Charge capacity of bare gold, Ppy, and PEDOT electrodes with applied charge density of $1.4 \mathrm{C} / \mathrm{cm}^{2}$ [31].

\begin{tabular}{cc}
\hline Electrodes & Charge capacity $(\mu \mathrm{C})$ \\
\hline Gold & $0.001 \pm 10^{-4}$ \\
Ppy & $2.3 \pm 0.5$ \\
PEDOT & $4.9 \pm 0.6$ \\
\hline
\end{tabular}

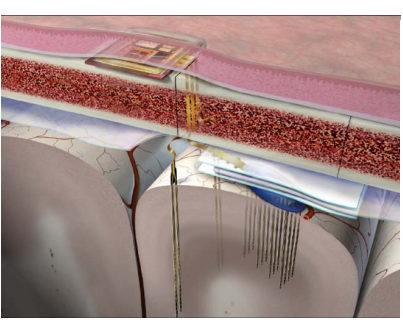

(a)

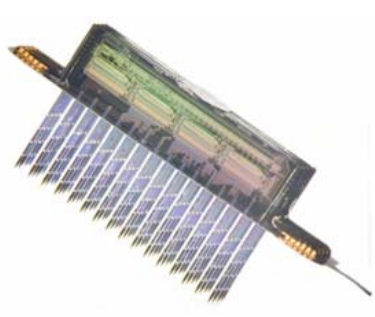

(b)
Figure 7. The Michigan cortical implant: (a) A 3D view of the microsystem which includes implanted electrodes with ribbon cables connecting them to an electronic device, including circuitry for amplification, spike detection, encoding, and wireless transmission of power and bidirectional data; (b) Four 64-site probes assembled into a 3D structure $[29]$.

trodes enables single-unit recording with high spatial resolution, as well as exciting the neurons by electrical stimulation. An array of electrodes with small surface areas and which penetrate to the cortex stimulate more localized regions of tissue [35]. Such microelectrode arrays can also be safely inserted into the brain [36]. To use microelectrode arrays for recording and stimulation, electrical and mechanical characteristics are important. Geometrical architecture is the parameter, which defines those characteristics in electrode arrays. Due to the Utah microelectrode arrays' architecture, they have been used extensively in neuroscience and clinical research. Figure 8 shows the two available architectures of Utah arrays, which count 100 microelectrodes with platinum-coated tips [37]. The Utah slanted electrode arrays provide 2D recording data from the slanted plane of the brain whereas the flat array provides $1 \mathrm{D}$ data. The issue with this type of arrays is that even the slanted array is still 2D instead of 3D. The latter microelectrodes are designed to be implanted in the cerebral cortex or peripheral nerves [1]. To facilitate charge transfer from the electrodes to neural tissue, the tips of the electrodes are coated with platinum (Pt), sputtered iridium oxide film (SIROF), titanium nitride (TiN), and soft conductive polymers such as Ppy and PEDOT. Pt is a noble metal and injects charge by both Faradaic reactions and double layer charging.

$\mathrm{Pt}$ charge inject capacity using $0.2 \mathrm{~ms}$ pulses is 50 $150 \mu \mathrm{C} / \mathrm{cm}^{2}$. Iridium oxide $\left(\mathrm{IrO}_{\mathrm{x}}\right)$ is using to coat the tips due to its ability to inject charge, its resistance to corro- 


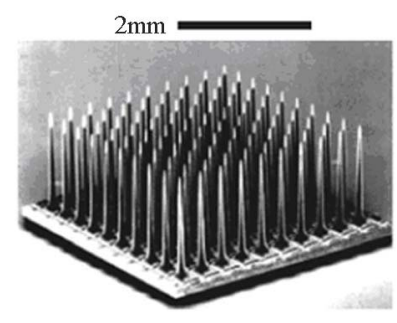

(a)

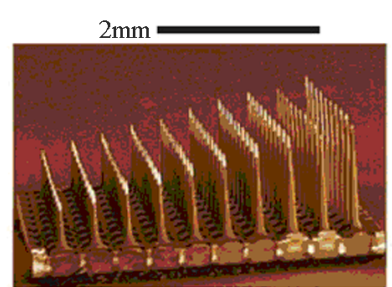

(b)
Figure 8. Utah silicon-based 100 microelectrode arrays: (a) 2D flat electrode array; (b) 2D slanted electrodes array [1, 37].

sion, and high conductivity. $\mathrm{IrO}_{\mathrm{x}}$ is a highly conductive oxide with bulk resistivity between 30 to $100 \mu \Omega \cdot \mathrm{cm}$. Using 0.2 ms pulses, the activated $\mathrm{IrO}_{\mathrm{x}}$ charge inject capacity will be $1 \mathrm{mC} / \mathrm{cm}^{2}$ [38]. TiN is another material found to be useful due to chemical stability and biocompatibility. Using $0.5 \mathrm{~ms}$ pulses provides $0.9 \mathrm{mC} / \mathrm{cm}^{2}$ charge injection capacity [39]. Table 3 shows the charge injection capacity of above materials. The protein adsorption of materials are being used for recording sites of implantable MEAs is another important factor to determine the stability of the implant. The absorbed protein layers on gold are thinner than other materials such as platinum and iridium. However, indium tin oxide might be a good alternative for coating [40].

Conductive polymers are now considered promising materials for chronic stimulation and recording due to their biocompatibility and large surface area which acts as a main factor to reduce the impedance of the electrodes. PEDOT nanotubes have a low impedance of $4 \mathrm{k} \Omega$ at $1 \mathrm{kHz}$, which increases the charge injection at the electrode-tissue interface.

Figures 9(a) and (b) shows the stability of the SIROFand Pt-coated electrodes in vitro over 90 days and a typical Bode plot as a function of frequency. The median impedance of Pt- and SIROF-coated electrodes were 125 $\pm 0.25 \mathrm{k} \Omega$ and $6.7 \pm 0.2 \mathrm{k} \Omega$ at $1 \mathrm{kHz}$, respectively. The impedance of uncoated silicon tips was $975 \pm 15 \mathrm{k} \Omega$ at 1 $\mathrm{kHz}$. A typical Bode plot presents the impedance of the Pt- and SIROF-coated Utah electrodes versus frequency. According to the plot, the impedance of the SIROF is lower than the Pt electrodes at all frequencies except at $105 \mathrm{~Hz}$, which is a high frequency [41].

\subsubsection{Metal-Based Microelectrode Array}

These microelectrodes arrays were fabricated by using a wire EDM technique associated with electrochemical steps [10]. The electrodes were made from stainless steel or titanium. After chemical etching to smooth the electrode surface, platinum was electro-deposited at the tips to facilitate charge transfer from electrode to neural tissue. To electrically insulate the electrodes parylene-C
Table 3. Charge injection capacity of coating materials [41].

\begin{tabular}{ccc}
\hline Electrodes & Pulse width $(\mathrm{ms})$ & Charge capacity $\left(\mu \mathrm{C} / \mathrm{cm}^{2}\right)$ \\
\hline $\mathrm{Pt}$ & 0.2 & $50-150$ \\
$\mathrm{IrO}_{\mathrm{x}}$ & 0.2 & 1000 \\
$\mathrm{TiN}$ & 0.5 & 900 \\
\hline
\end{tabular}

was chosen as an insulator due to its biocompatibility. Electrodes were insulated from each other by epoxy [11]. Figure 10 shows a general view of these fabricated electrodes. This microelectrode array was developed to be assembled with integrated circuits on a thin substrate. [42]. The main drawback of these electrodes is that they are not compatible with integrated circuit manufacturing techniques. The impedance of the electrodes was obtained from the impedance curves for each electrode using an impedance analyser. Measurements were performed at $25^{\circ} \mathrm{C}$ in $0.9 \%$ saline; 43 out of 64 electrodes had an average impedance of $78 \mathrm{k} \Omega$, and 21 of electrodes had an average impedance of $5.7 \mathrm{M} \Omega$.

\subsection{Flexible Array}

A variety of MEAs has been developed for recording and monitoring neural activities; however, most MEAs are based on a rigid substrate and cause neural damage and inflammation at the implant site for intra-cortical implantation. Flexible arrays allow electrodes to fit on the surface of the brain to record and monitor in a less invasive way. Flexible, polyamide-based microelectrodes are used for chronic neural recording because they provides high mechanical flexibility, good biocompatibility, high resistance to solvents, and are a strong addition to metal oxides [43]. Different polymers such as polyimide and parylene are used as a structural substrate. Parylene-based MEAs are extremely flexible, providing highly conformal coverage of the muscle surface and a stable electrical contact, which results in an improved SNR [44]. Recently, flexible CNT microelectrodes have not only improved electrode impedance and charge-transfer capacity but also-when the CNTs are grown directly on the polyimide substrate - their adhesion to the substrate is improved [45].

Flexible parylene-based microelectrode arrays for recordings are shown in Figure 11.

A new parylene-based multi-sided MEA with electrode sites at the top-side, back-side, and edge has been presented for neural recording and passive drug delivery. This feature creates the smallest footprint $\left(85 \mu \mathrm{m}^{2}\right)$ to date of a functional recording electrode. Gold microelectrodes have an average impedance of $6.0 \mathrm{M} \Omega$ at $1 \mathrm{kHz}$ which, by using PEDOT electropolymerization, reduce the impedance to $210 \mathrm{k} \Omega$. In fact, by increasing the electrochemical area, PEDOT polymerization decreases the 


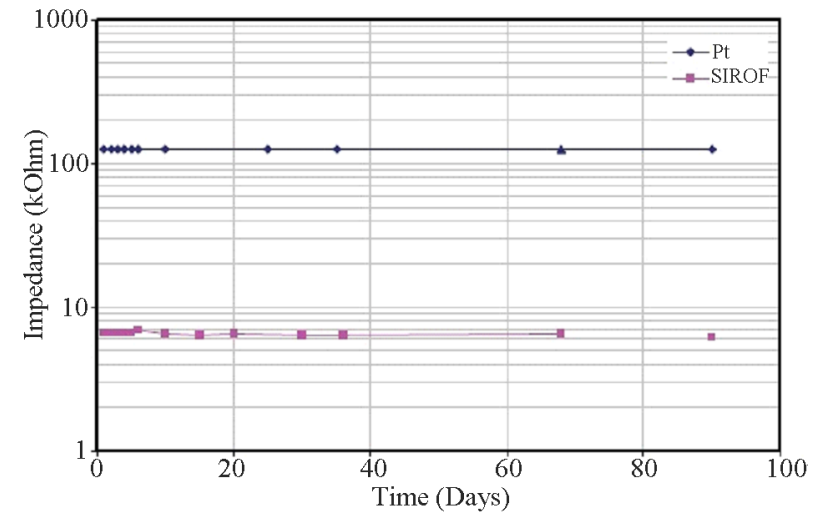

(a)

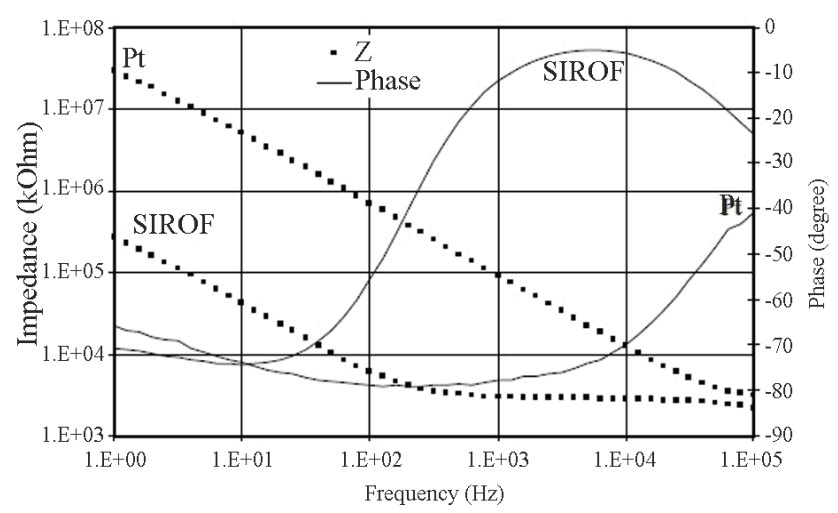

(b)

Figure 9. Stability of Pt- and SIROF-coated electrodes in vitro over 90 days; (b) The Bode plot presents the electrode impedance versus frequency of Pt- and SIROF-coated Utah electrodes [41].

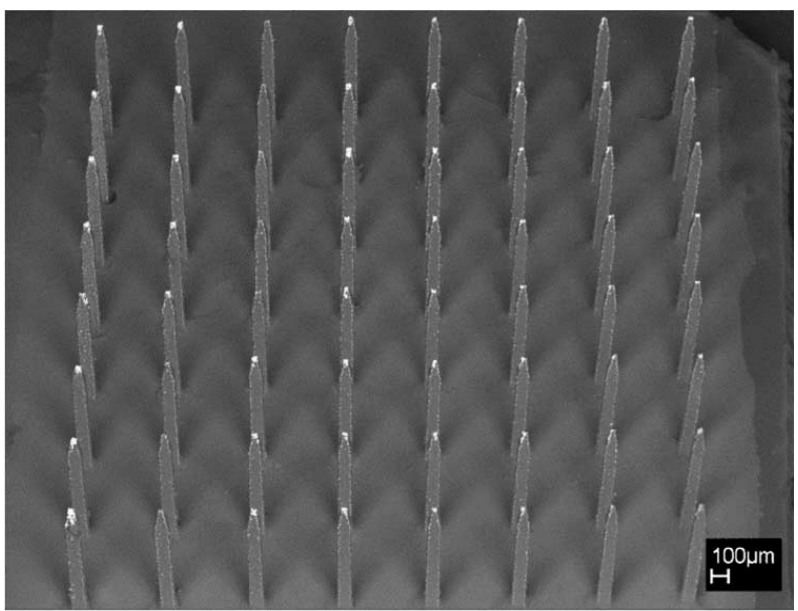

(a)

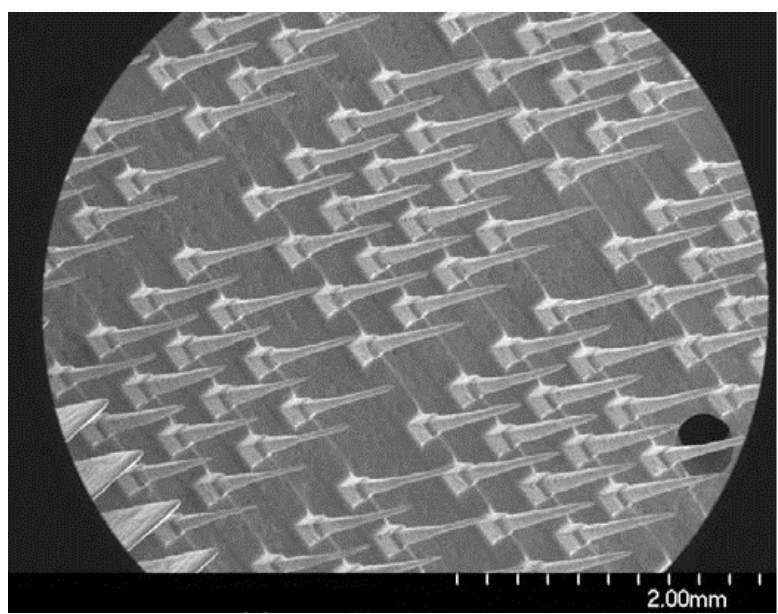

(b)

Figure 10. Metallic-based microelectrode arrays using an EDM technique: (a) SEM image of a parylene-coated assembly, consisting of platinum-coated electrode tips; (b) Stainless steel electrode arrays after electrochemical polishing [10,11].

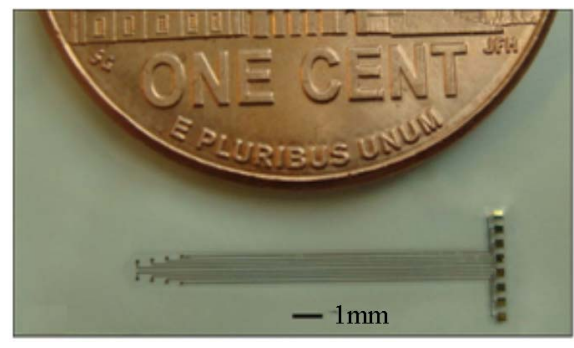

(a)

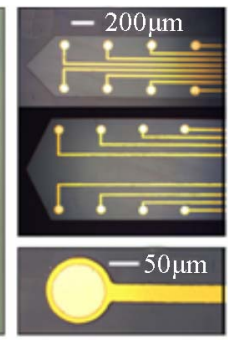

(b)
Figure 11. Flexible parylene-based electrodes: (a) Global view of eight microelectrodes; (b) Photomicrograph picture of microelectrodes with two different spaces between the recording sites; (c) Photomicrograph picture of a single recording site that includes a gold pad and a parylene-insulated region (dark yellow) [44].

overall impedance. A different design of novel pary-lene-based microelectrodes for recording, passive drug delivery, and cell delivery are shown in Figure 12 [34].
Table 4 shows the impedance of the gold and PEDOT-coated recording sites at the surface and the edge.

Flexible surface electrodes provoke less tissue damage because the surgeon may avoid penetrating neural tissues; however, surface electrodes have low sensitivity and selectivity. In addition, because of its flexible nature, there is difficulty inserting electrodes into the nervous tissue.

\section{Electrode-Tissue Interface}

Electrical coupling and biocompatibility are the most important issues at electrode-tissue interfaces. While electrical coupling is determined by the impedance at the interface, biocompatibility is influenced by the materials at the recording or stimulation site [46]. Low impedance causes low noise and is obtained with a large surface area. A new approach to this issue is using nanomaterials such as semiconductor quantum dots [47], metallic nanoparticles [48], metallic or semiconductor nanowires, nanos- 


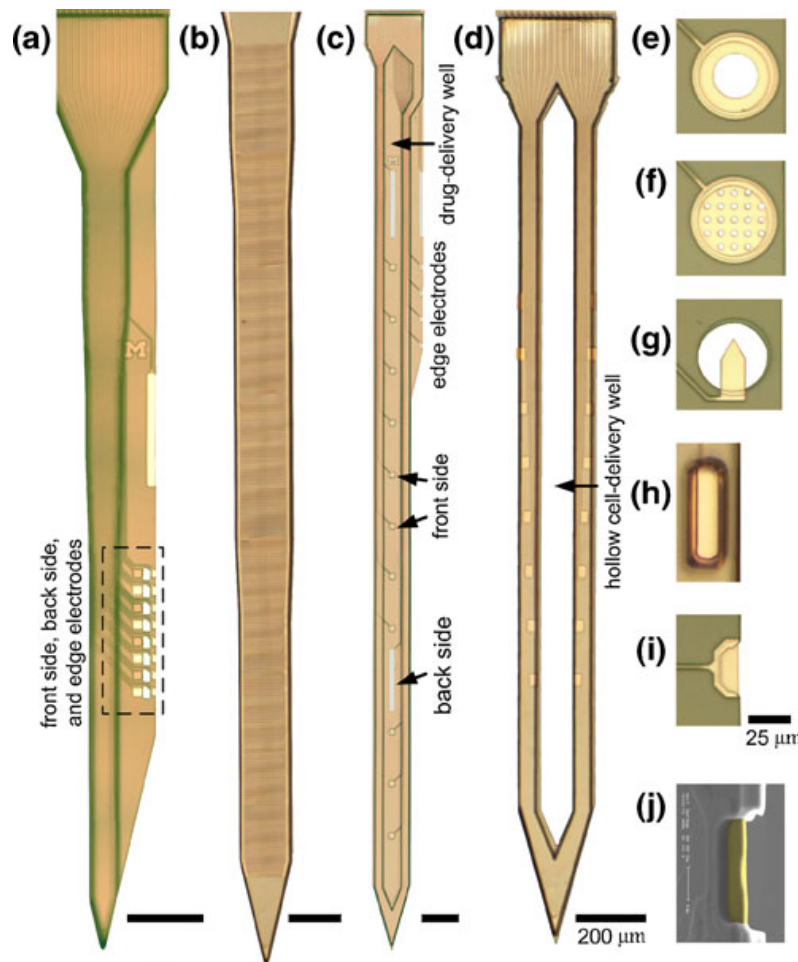

Figure 12. Different designs of multi-sided microelectrode: (a) Front-side, back-side, and edge designs of electrodes with 45-micron spacing; (b) Open-channel design for passive drug delivery; (c) Hollow-channel neural probe for cell-based therapies; (d) Ring, sieve, cantilever, reservoir, edge, PEDOT-coated edge electrodes [34].

tructured conductive polymers [49], carbon nanofibers [50], or carbon CNTs that are characterized by excellent electrical [51], mechanical, and chemical [52] properties for neural interfaces. Electronic properties of the nanomaterials are match with the charge transport requirements of cellular interfacing besides the mechanical, and the chemical properties of nanomaterials are critical for long-term implants. Nanomaterials play a significant role in improving electrode domains by increasing the sensitivity and selectivity, improving response time, and making the electrodes biocompatible with the biological environment. Due to their rich electronic properties, high aspect ratio, excellent chemical stability, high mechanical strength, and intrinsic large surface area, CNTs are one of the best options for the active site of electrodes [53].

Other promising materials to coat nerve electrodes are polymers with properties of their modification with biomolecules to improve biocompatibility and create a biologically active electrode-tissue interface. PEDOT is an electrical conductive polymer and the most promising material to coat electrodes $[54,55]$.

Implanted electrodes exhibit a short, effective lifetime due to degradation of signal transmission at the tissue-electrode interface [56]. Optimum electrodes,
Table 4. Electrode sites impedance [34].

\begin{tabular}{ccc}
\hline & $\begin{array}{c}\text { Au site, } \\
\mathrm{N}=48\end{array}$ & PEDOT-PSS $^{1}$ site, $\mathrm{N}=33$ \\
\hline Planar $17 \times 17 \mu \mathrm{m}$ & $5.4 \pm 3.7 \mathrm{M} \Omega$ & $240 \pm 110 \mathrm{k} \Omega$ \\
Edge $17 \times 5 \times 2 \mu \mathrm{m}$ & $6.0 \pm 3.1 \mathrm{M} \Omega$ & $210 \pm 64 \mathrm{k} \Omega$ \\
\hline
\end{tabular}

${ }^{1}$ PSS: Polystyrene Sulfonate.

which implant in neural tissue, should cover two important characteristics: long-term tissue compatibility and fast response to signals [57]. Long-term tissue compatibility is related to a tissue response to a foreign body. This response results in glial activation. Microglial cells, which integrate the intrinsic brain defence system, are very sensitive to variations in extracellular ionic concentrations and form an insulating interface that prevents signal conduction or charge injection for long-term implantation [58]. Local activation of glial cells causes electrode encapsulation due to chronic electrode implant and it increases the electrical impedance of the recording site [59]. Different methods are being used to prevent cellular encapsulation and electrode failure. Surface modification of electrodes can improve the interaction between neural cells and implants. L1, a neural adhesion molecule, is one of the coating materials that may increase the biocompatibility of neural probes and promote electro-detissue interface.

\section{Comparison of Microelectrode Technologies}

Findings to date demonstrate different methods for recording and stimulating neuron activities; however, all these methods have some drawbacks. The tables (Tables 5-7) show a brief comparison of various techniques for fabricating MEAs.

\section{Design and Microfabrication of MEAs}

As described above, various types of MEAs exist; these are silicon-based, treated with dedicated electrochemical steps; however, these types of microelectrode arrays, fabricated by micromachining techniques, still lack the required 3D, high-density and low-impedance, necessary for ETCs and required by implantable stimulators. By focusing on these aspects the ultimate purpose of introducing new microelectrodes is to elaborate effective MEAs with selectivity, sensitivity, biocompatibility, good electric characteristics, and long-term chemical and recording stability. To achieve this goal, a silicon-based micromachined method was chosen to make the new microelectrodes due to compatibility with integrated circuit manufacturing techniques and a high degree of selectivity in stimulation and recording. Front-side diced electrodes were etched after backside dicing, glassing, and metallization. As a result, we obtained electrode ar- 
Table 5. Advantages and drawbacks of MEA fabrication methods [6,9,24,60].

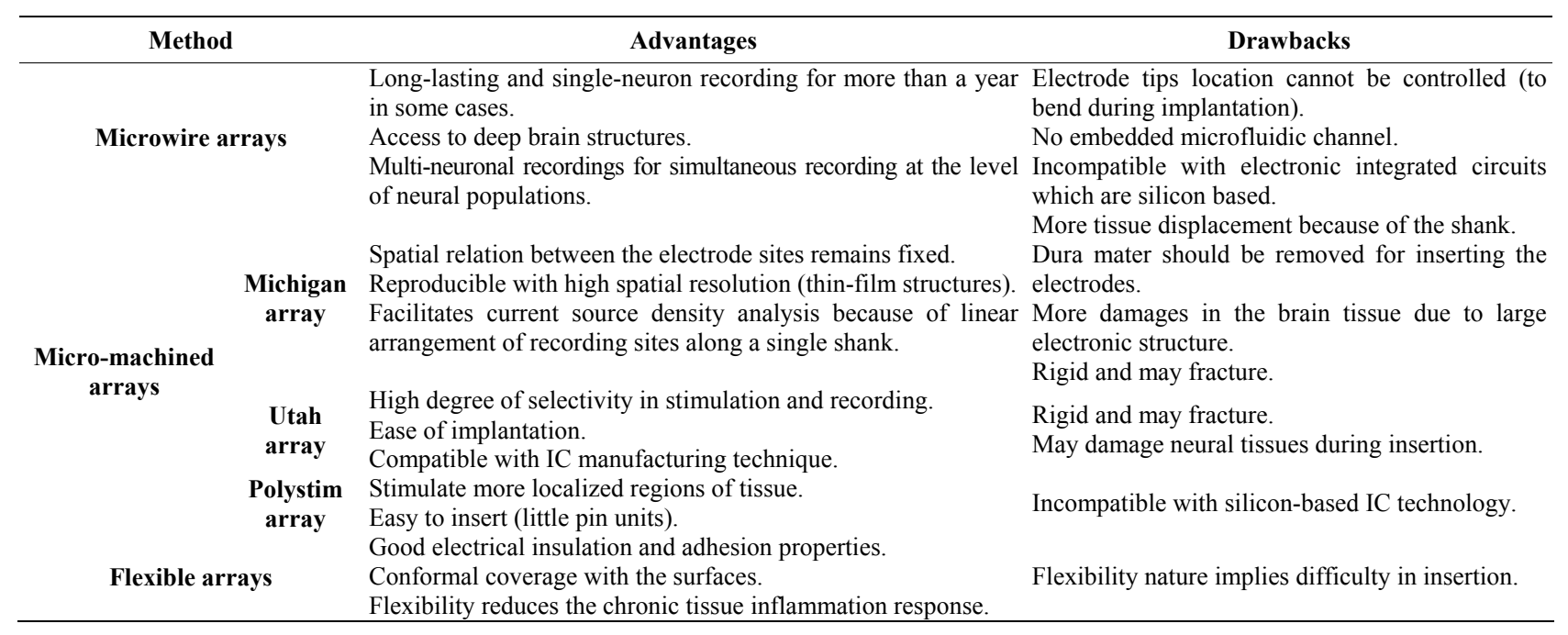

Table 6. Characterization of MEA technologies [31,41].

\begin{tabular}{|c|c|c|c|c|c|}
\hline & MEAs & & MEAs sites & Mean charge density $\left(\mathrm{mC} / \mathrm{cm}^{2}\right)$ & Mean Impedance- $\mathrm{Z}_{1 \mathrm{kHz}}(\mathrm{k} \Omega)$ \\
\hline \multirow{4}{*}{ Silicon-based } & \multirow{2}{*}{ Utah array } & SIROF & 96 & 2 & $6.1 \pm 0.2$ \\
\hline & & Pt & 96 & 0.3 & $125 \pm 0.25$ \\
\hline & \multirow{2}{*}{ Michigan array } & Polypyrrole & \multirow{2}{*}{64} & $19.5 \pm 2.1$ & $184 \pm 5.3$ \\
\hline & & PEDOT & & $2.5 \pm 1.4$ & $392 \pm 6.2$ \\
\hline & TDT $^{1}$ Microwires & & 48 & $5.10 \pm 0.40$ & $19.9 \pm 0.82$ \\
\hline
\end{tabular}

${ }^{1}$ TDT $=$ Tucker Davis Technologies.

Table 7. Comparison of microelectrode structure [43,61-63].

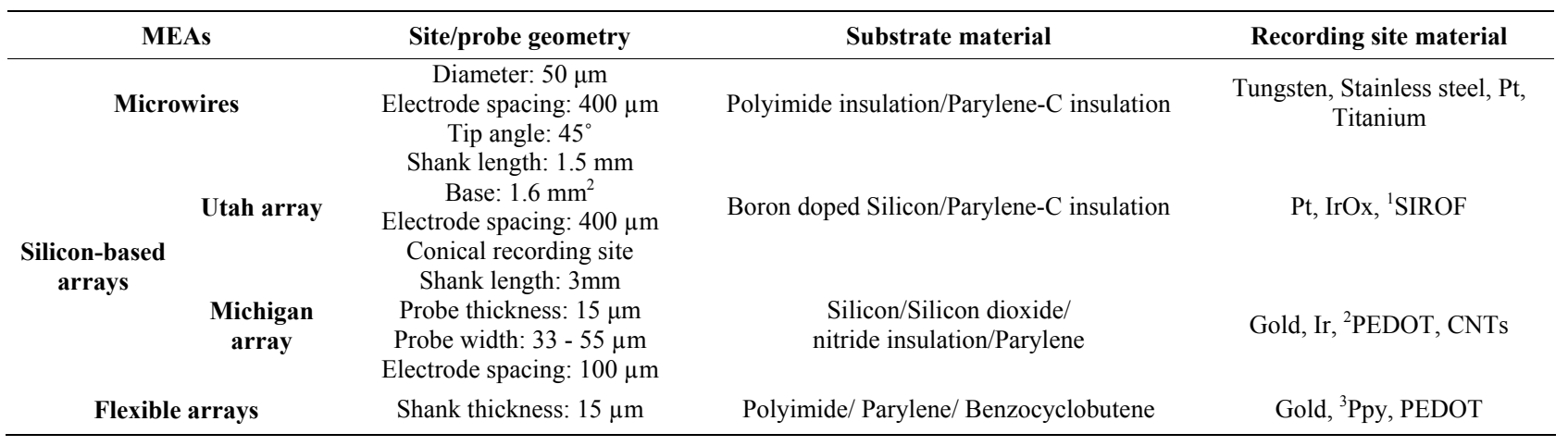

${ }^{1}$ SIROF: Sputtered Iridium Oxide Films; ${ }^{2}$ PEDOT: poly (3, or 4-ethylenedioxythiophene); ${ }^{3}$ Ppy: Polypyrrole.

rays with variable heights of $1.45,1.55$, and $1.65 \mathrm{~mm}$. The thickness of the electrodes was $200 \mu \mathrm{m}$ at the base and $5 \mu \mathrm{m}$ at the tip with $98 \mu \mathrm{m}$ spacing. Figures 13(a) and (b) shows the preliminary result of fabricated MEAs and depict the desired 3D electrode array. We are fabricating 3D high-density silicon-based MEAs. Such 3D silicon-based microelectrodes in an array will allow not only recording from different depths of the brain but cause less tissue damage during insertion.

To enhance the high-selective, low-impedance, and high signal-to-noise ratio necessary for ETCs, fabricated silicon probes capable of simultaneously recording and stimulating from neuronal populations will be coated with various conductive polymers and CNTs.

\section{Discussion}

The emerging field of neuroprostheses that incorporates BMIs is being developed to solve nervous system disorders that people suffering from. To date the technology for creating MEAs capable of restoring lost neural function is still in progress. Clearly, much work still needs to be done to accept a standard model of MEAs for clinical purposes. Electrical and mechanical characteristics of 


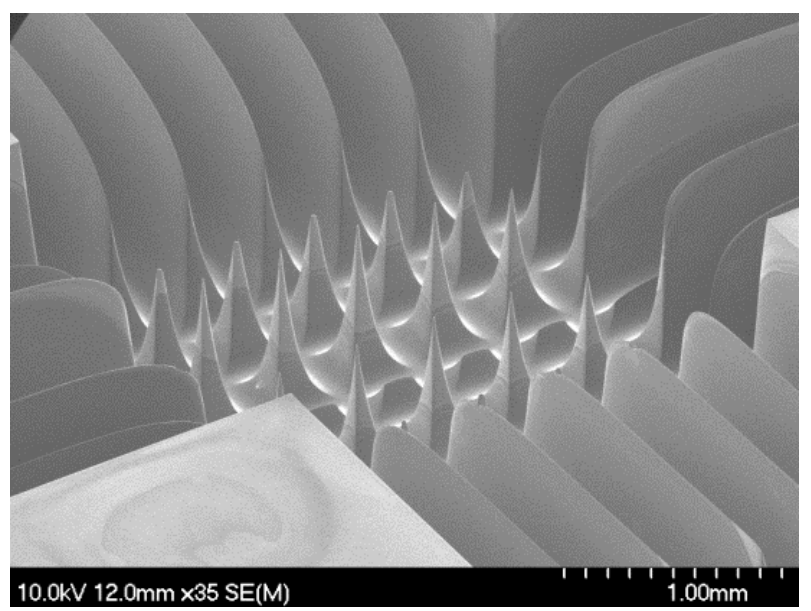

(a)

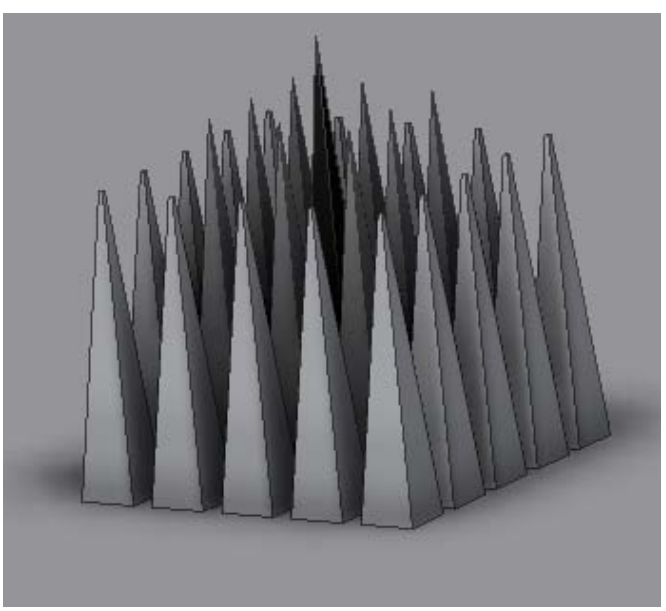

(b)

Figure 13. Microelectrode arrays: (a) SEM image of the MEAs; (b) Schematic of proposed 3D MEAs.

electrode arrays are important for stimulation or recording. Geometrical characteristics of the electrodes in an array will affect the electrical and mechanical properties. Mechanical conformity minimizes tissue damage during insertion. 3D electrodes with variable heights of the electrodes in an array will provide more contacts between the electrodes and targeted neural tissue. More importantly, the novel geometry will allow not only recording from different depths of the brain but will cause less tissue damage during insertion. To achieve high-density electrode arrays in this proposed structure, the distance between electrodes is decreased. Therefore, the density of pins will be higher, which will allow us to selectively stimulate in various sites in the $3 \mathrm{D}$ area of the MEAs. To achieve electrodes with higher selectivity and less tissue damage during penetration into the tissue, thinner and sharper electrodes are desired. Besides, small tip exposures increase the electrode impedance. To improve neuronal signals, the impedance of the electrode should be low. To achieve this goal, the tips of the electrodes will be coated with conductive polymers and CNTs to improve the recording and stimulation characteristics of the neural electrodes. The coating enhances electrical properties by decreasing electrode impedance and increasing the charge transfer.

\section{Acknowledgements}

We gratefully acknowledge the financial support from the Natural Sciences and Engineering Research Council of Canada (NSERC) and the Canada Research Chair in smart medical devices, and design tools and fabrication resources from CMC Microsystems.

\section{REFERENCES}

[1] R. A. Normann, “Technology Insight: Future Neuropros- thetic Therapies for Disorders of the Nervous System," Nature Clinical Practice Neurology, Vol. 3, No. 8, 2007, pp. 444-452. doi:10.1038/ncpneuro0556

[2] E. Patrick, "Design, Fabrication, and Characterization of Microelectrodes for Brain-machine Interfaces," University of Florida, Gainesville, 2010.

[3] J. W. Judy, "Microelectrode Technologies for Neuroengineered Systems," Proceedings of the 25th Annual International Conference of the IEEE Engineering in Medicine and Biology Society, Cancun, 17-21 September 2003, pp. 3806-3809.

[4] E. W. Keefer, B. R. Botterman, M. I. Romero, A. F. Rossi and G. W. Gross, "Carbon Nanotube Coating Improves Neuronal Recordings," Nature Nanotechnology, Vol. 3, No. 7, 2008, pp. 434-439. doi:10.1038/nnano.2008.174

[5] Y. F. Rui, J. Q. Liu, B. Yang, K. Y. Li and C. S. Yang, "Parylene-Based Implantable Platinum-Black Coated Wire Microelectrode for Orbicularis Oculi Muscle Electrical Stimulation," Biomedical Microdevices, Vol. 14, No. 2, 2012, pp. 367-373. doi:10.1007/s10544-011-9613-8

[6] K. C. Cheung, "Implantable Microscale Neural Interfaces," Biomedical Microdevices, Vol. 9, No. 6, 2007, pp. 923938. doi:10.1007/s10544-006-9045-Z

[7] F. Strumwasser, "Long-Term Recording from Single Neurons in Brain of Unrestrained Mammals," Science, Vol. 127, No. 3296, 1958, pp. 469-470. doi:10.1126/science.127.3296.469

[8] K. L. Drake, K. D. Wise, J. Farraye, D. J. Anderson and S. L. BeMent, "Performance of Planar Multisite Microprobes in Recording Extracellular Single-Unit Intracortical Activity," IEEE Transactions on Bio-Medical Engineering, Vol. 35, No. 9, 1988, pp. 719-732. doi: $10.1109 / 10.7273$

[9] P. K. Campbell, K. E. Jones, R. J. Huber, K. W. Horch and R. A. Normann, "A Silicon-Based, Three-Dimensional Neural Interface: Manufacturing Processes for an Intracortical Electrode Array," IEEE Transactions on BioMedical Engineering, Vol. 38, No. 8, 1991, pp. 758-768. doi: $10.1109 / 10.83588$ 
[10] T. A. Fofonoff, S. M. Martel, N. G. Hatsopoulos, J. P. Donoghue and I. W. Hunter, "Microelectrode Array Fabrication by Electrical Discharge Machining and Chemical Etching," IEEE Transactions on Bio-Medical Engineering, Vol. 51, No. 6, 2004, pp. 890-895. doi:10.1109/TBME.2004.826679

[11] A. E. Ayoub, B. Gosselin and M. Sawan, "A Microsystem Integration Platform Dedicated to Build Multi-ChipNeural Interfaces," Conference Proceedings: Annual International Conference of the IEEE Engineering in Medicine and Biology Society, Lyon, 23-26 August 2007, pp. 6605-6608.

[12] J. Subbaroyan, D. C. Martin and D. R. Kipke, "A FiniteElement Model of the Mechanical Effects of Implantable Microelectrodes in the Cerebral Cortex," Journal of Neural Engineering, Vol. 2, No. 4, 2005, pp. 103-113. doi:10.1088/1741-2560/2/4/006

[13] M. P. Ward, P. Rajdev, C. Ellison and P. P. Irazoqui, "Toward a Comparison of Microelectrodes for Acute and Chronic Recordings," Brain Research, Vol. 1282, 2009, pp. 183-200. doi:10.1016/j.brainres.2009.05.052

[14] R. A. Green, "Unauthorised Development and Seismic Hazard Vulnerability: A Study of Squatters and Engineers in Istanbul, Turkey," Disasters, Vol. 32, No. 3, 2008, pp. 358-376. doi:10.1111/j.1467-7717.2008.01044.x

[15] M. Verzeano and K. Negishi, "Neuronal Activity in Cortical and Thalamic Networks," The Journal of General Physiology, Vol. 43, No. 6, 1960, pp. 177-195. doi:10.1085/igp.43.6.177

[16] B. Franklin, "An Account of the Effects of Electricity in Paralytic Cases. In a Letter to John Pringle, MDFRS from Benjamin Franklin, Esq; FRS," Philosophical Transactions (1683-1775), Vol. 50, 1757, pp. 481-483.

[17] G. E. Loeb, R. A. Peck and J. Martyniuk, "Toward the Ultimate Metal Microelectrode," Journal of Neuroscience Methods, Vol. 63, No. 1, 1995, pp. 175-183. doi:10.1016/0165-0270(95)00107-7

[18] D. A. Robinson, "The Electrical Properties of Metal Microelectrodes," Proceedings of the IEEE, Vol. 56, No. 6, 1968, pp. 1065-1071. doi:10.1109/PROC.1968.6458

[19] D. H. Hubel, "Tungsten Microelectrode for Recording from Single Units," Science, Vol. 125, No. 3247, 1957, pp. 549-550. doi:10.1126/science.125.3247.549

[20] R. F. Thompson and M. M. Patterson, "Bioelectric Recording Techniques," Academic Press, Waltham, 1974.

[21] J. K. Chapin, "Using Multi-Neuron Population Recordings for Neural Prosthetics," Nature Neuroscience, Vol. 7, No. 5, 2004, pp. 452-455. doi:10.1038/nn1234

[22] G. W. Gross, W. Y. Wen and J. W. Lin, "Transparent Indium-Tin Oxide Electrode Patterns for Extracellular, Multisite Recording in Neuronal Cultures," Journal of Neuroscience Methods, Vol. 15, No. 3, 1985, pp. 243252. doi:10.1016/0165-0270(85)90105-0

[23] R. A. Green, C. M. Williams, N. H. Lovell and L. A. PooleWarren, "Novel Neural Interface for Implant Electrodes: Improving Electroactivity of Polypyrrole through MWNT Incorporation," Journal of Materials Science. Materials in Medicine, Vol. 19, No. 4, 2008, pp. 1625-1629.

\section{doi:10.1007/s10856-008-3376-7}

[24] K. D. Wise, J. B. Angell and A. Starr, "An IntegratedCircuit Approach to Extracellular Microelectrodes," IEEE Transactions on Biomedical Engineering, Vol. 17, No. 3, 1970, pp. 238-247. doi:10.1109/TBME.1970.4502738

[25] K. Wise and J. Angell, "A Microprobe with Integrated Amplifiers for Neurophysiology," IEEE International Solid-State Circuits Conference. Digest of Technical Papers, Philadelphia, 17-19 February 1971, pp. 100-101.

[26] G. S. Brindley and W. S. Lewin, "The Sensations Produced by Electrical Stimulation of the Visual Cortex," The Journal of Physiology, Vol. 196, No. 2, 1968, pp. 479-493.

[27] W. H. Dobelle, M. G. Mladejovsky and J. P. Girvin, “Artifical Vision for the Blind: Electrical Stimulation of Visual Cortex Offers Hope for a Functional Prosthesis," Science, Vol. 183, No. 4123, 1974, pp. 440-444.

doi:10.1126/science. 183.4123 .440

[28] R. J. Vetter, J. C. Williams, J. F. Hetke, E. A. Nunamaker and D. R. Kipke, "Chronic Neural Recording Using Silicon-Substrate Microelectrode Arrays Implanted in Cerebral Cortex," IEEE Transactions on Bio-Medical Engineering, Vol. 51, No. 6, 2004, pp. 896-904. doi:10.1109/TBME.2004.826680

[29] K. D. Wise, A. M. Sodagar, Y. Yao, M. N. Gulari, G. E. Perlin and K. Najafi, "Microelectrodes, Microelectronics, and Implantable Neural Microsystems," Proceedings of the IEEE, Vol. 96, No. 7, 2008, pp. 1184-1202. doi:10.1109/JPROC.2008.922564

[30] K. D. Wise, D. J. Anderson, J. F. Hetke, D. R. Kipke and K. Najafi, "Wireless Implantable Microsystems: HighDensity Electronic Interfaces to the Nervous System," Proceedings of the IEEE, Vol. 92, No. 1, 2004, pp. 76-97. doi:10.1109/JPROC.2003.820544

[31] M. R. Abidian and D. C. Martin, "Experimental and Theoretical Characterization of implantable Neural Microelectrodes Modified with Conducting Polymer Nanotubes," Biomaterials, Vol. 29, No. 9, 2008, pp. 1273-1283. doi:10.1016/j.biomaterials.2007.11.022

[32] T. Gabay, M. Ben-David, I. Kalifa, R. Sorkin, Z. R. Abrams, E. Ben-Jacob and Y. Hanein, "Electro-Chemical and Biological Properties of Carbon Nanotube Based MultiElectrode Arrays," Nanotechnology, Vol. 18, No. 3, 2007, Article ID: 035201. doi:10.1088/0957-4484/18/3/035201

[33] S. M. E. Merriam, "A Three-Dimensional Bidirectional Interface for Neural Mapping Studies," Solid State Electronics Lab, University of Michigan, Michigan, 2010.

[34] J. P. Seymour, N. B. Langhals, D. J. Anderson and D. R. Kipke, "Novel Multi-Sided, Microelectrode Arrays for Implantable Neural Applications," Biomedical Microdevices, Vol. 13, No. 3, 2011, pp. 441-451. doi:10.1007/s10544-011-9512-z

[35] S. F. Ronner and B. G. Lee, "Excitation of Visual Cortex Neurons by Local Intracortical Microstimulation," Experimental Neurology, Vol. 81, No. 2, 1983, pp. 376-395. doi:10.1016/0014-4886(83)90270-4

[36] R. A. Normann, E. M. Maynard, P. J. Rousche and D. J. 
Warren, "A Neural Interface for a Cortical Vision Prosthesis," Vision Research, Vol. 39, No. 15, 1999, pp. 2577 2587. doi:10.1016/S0042-6989(99)00040-1

[37] R. Bhandari, S. Negi and F. Solzbacher, "Wafer-Scale Fabrication of Penetrating Neural Microelectrode Arrays," Biomedical Microdevices, Vol. 12, No. 5, 2010, pp. 797-807. doi:10.1007/s10544-010-9434-1

[38] S. F. Cogan, P. R. Troyk, J. Ehrlich and T. D. Plante, "In Vitro Comparison of the Charge-Injection Limits of Activated Iridium Oxide (AIROF) and Platinum-Iridium Microelectrodes," IEEE Transactions on Biomedical Engineering, Vol. 52, No. 9, 2005, pp. 1612-1614. doi:10.1109/TBME.2005.851503

[39] J. D. Weiland, D. J. Anderson and M. S. Humayun, "In Vitro Electrical Properties for Iridium Oxide Versus Titanium Nitride Stimulating Electrodes," IEEE Transactions on Biomedical Engineering, Vol. 49, No. 12, 2002, pp. 1574-1579. doi:10.1109/TBME.2002.805487

[40] J. Selvakumaran, J. L. Keddie, D. J. Ewins and M. P. Hughes, "Protein Adsorption on Materials for Recording Sites on Implantable Microelectrodes," Journal of Materials Science. Materials in Medicine, Vol. 19, No. 1, 2008, pp. 143-151.

[41] S. Negi, R. Bhandari, L. Rieth and F. Solzbacher, "In Vitro Comparison of Sputtered Iridium Oxide and Platinum-Coated Neural Implantable Microelectrode Arrays," Biomedical Materials, Vol. 5, No. 1, 2010, Article ID: 015007. doi:10.1088/1748-6041/5/1/015007

[42] J. B. Kirkpatrick, M. L. Higgins, J. H. Lucas and G. W. Gross, "In Vitro Simulation of Neural Trauma by Laser," Journal of Neuropathology and Experimental Neurology, Vol. 44, No. 3, 1985, pp. 268-284. doi:10.1097/00005072-198505000-00005

[43] K. C. Cheung, P. Renaud, H. Tanila and K. Djupsund, "Flexible Polyimide Microelectrode Array for in Vivo Recordings and Current Source Density Analysis," Biosensors \& Bioelectronics, Vol. 22, No. 8, 2007, pp. 17831790. doi:10.1016/j.bios.2006.08.035

[44] C. Metallo, R. D. White and B. A. Trimmer, "Flexible Parylene-Based Microelectrode Arrays for High Resolution EMG Recordings in Freely Moving Small Animals," Journal of Neuroscience Methods, Vol. 195, No. 2, 2011, pp. 176-184.doi:10.1016/j.jneumeth.2010.12.005

[45] Y. C. Chen, H. L. Hsu, Y. T. Lee, H. C. Su, S. J. Yen, C. H. Chen, W. L. Hsu, T. R. Yew, S. R. Yeh, D. J. Yao, Y. C. Chang and H. Chen, "An Active, Flexible Carbon Nanotube Microelectrode Array for Recording Electrocorticograms," Journal of Neural Engineering, Vol. 8, No. 3, 2011, Article ID: 034001. doi:10.1088/1741-2560/8/3/034001

[46] C. Nick, R. Joshi, J. J. Schneider and C. Thielemann, "Three-Dimensional Carbon Nanotube Electrodes for Extracellular Recording of Cardiac Myocytes," Biointerphases, Vol. 7, 2012, p. 58. doi:10.1007/s13758-012-0058-2

[47] R. A. Green, S. Baek, L. A. Poole-Warren and P. J. Martens, "Conducting Polymer-Hydrogels for Medical Electrode Applications," Science and Technology of Advanced Materials, Vol. 11, No. 1, 2010, Article ID: 014107.

\section{doi:10.1088/1468-6996/11/1/014107}

[48] J. P. Frampton, M. R. Hynd, M. L. Shuler and W. Shain, "Effects of Glial Cells on Electrode Impedance Recorded from Neural Prosthetic Devices in Vitro," Annals of Biomedical Engineering, Vol. 38, No. 3, 2010, pp. 10311047. doi:10.1007/s10439-010-9911-y

[49] A. Prasad and J. C. Sanchez, "Quantifying Long-Term Microelectrode Array Functionality Using Chronic in Vivo Impedance Testing," Journal of Neural Engineering, Vol. 9, No. 2, 2012, Article ID: 026028. doi:10.1088/1741-2560/9/2/026028

[50] S. F. Cogan, "Neural Stimulation and Recording Electrodes," Annual Review of Biomedical Engineering, Vol. 10, 2008, pp. 275-309. doi:10.1146/annurev.bioeng.10.061807.160518

[51] J. O. Winter, M. Gokhale, R. J. Jensen, S. F. Cogan and J. F. Rizzo 3rd, "Tissue Engineering Applied to the Retinal Prosthesis: Neurotrophin-Eluting Polymeric Hydrogel Coatings," Materials Science \& Engineering. C, Materials for Biological Applications, Vol. 28, No. 3, 2008, pp. 448-453.

[52] X. Y. Cui, L. Li, Y. Y. An and G. W. Lu, "Changes in the Contents of Glycogen and Lactate in the Brain and Blood during Hypoxic Preconditioning," Acta Physiologica Sinica, Vol. 53, No. 4, 2001, pp. 325-328.

[53] G. Santhanam, S. I. Ryu, M. Y. Byron, A. Afshar and K. V. Shenoy, "A High-Performance Brain-Computer Interface," Nature, Vol. 442, No. 7099, 2006, pp. 195-198.

[54] X. Cui, V. A. Lee, Y. Raphael, J. A. Wiler, J. F. Hetke, D. J. Anderson and D. C. Martin, "Surface Modification of Neural Recording Electrodes with Conducting Polymer/ Biomolecule Blends," Journal of Biomedical Materials Research, Vol. 56, No. 2, 2001, pp. 261-272. doi:10.1002/1097-4636(200108)56:2<261::AID-JBM109 4>3.0.CO;2-I

[55] X. Y. Cui and D. C. Martin, "Electrochemical Deposition and Characterization of Poly(3,4-Ethylenedioxythiophene) on Neural Microelectrode Arrays," Sensors and Actuators B: Chemical, Vol. 89, No. 1-2, 2003, pp. 92-102. doi:10.1016/S0925-4005(02)00448-3

[56] P. A. Forcelli, C. T. Sweeney, A. D. Kammerich, B. C. Lee, L. H. Rubinson, Y. P. Kayinamura, K. Gale and J. F. Rubinson, "Histocompatibility and in Vivo Signal Throughput for PEDOT, PEDOP, P3MT, and Polycarbazole Electrodes," Journal of Biomedical Materials Research. Part A, Vol. 100, No. 12, 2012, pp. 3455-3462.

[57] X. Cui, H. Shin, C. Song, W. Laosinchai, Y. Amano and R. M. Brown Jr., "A Putative Plant Homolog of the Yeast beta-1,3-Glucan Synthase Subunit FKS1 from Cotton (Gossypium hirsutum L.) Fibers," Planta, Vol. 213, No. 2, 2001, pp. 223-230. doi:10.1007/s004250000496

[58] A. S. Dickey, A. Suminski, Y. Amit and N. G. Hatsopoulos, "Single-Unit Stability Using Chronically Implanted Multielectrode Arrays," Journal of Neurophysiology, Vol. 102, No. 2, 2009, pp. 1331-1339. doi:10.1152/jn. 90920.2008

[59] M. A. Lebedev and M. A. Nicolelis, "Brain-Machine Interfaces: Past, Present and Future," Trends in Neurosciences, Vol. 29, No. 9, 2006, pp. 536-546. 
doi:10.1016/j.tins.2006.07.004

[60] C. L. Kolarcik, D. Bourbeau, E. Azemi, E. Rost, L. Zhang, C. F. Lagenaur, D. J. Weber and X. T. Cui, "In Vivo Effects of L1 Coating on Inflammation and Neuronal Health at the Electrode-Tissue Interface in Rat Spinal Cord and Dorsal Root Ganglion," Acta Biomaterialia, Vol. 8, No. 10, 2012, pp. 3561-3575. doi:10.1016/j.actbio.2012.06.034

[61] T. Stieglitz, H. Beutel, M. Schuettler and J. U. Meyer, "Micromachined, Polyimide-Based Devices for Flexible
Neural Interfaces," Biomedical Microdevices, Vol. 2, No. 4, 2000, pp. 283-294. doi:10.1023/A:1009955222114

[62] M. A. L. Nicolelis, "Brain-Machine Interfaces to Restore Motor Function and Probe Neural Circuits," Nature Reviews Neuroscience, Vol. 4, No. 5, 2003, pp. 417-422.

[63] D. R. Kipke, "Implantable Neural Probe Systems for Cortical Neuroprostheses," Conference Proceedings: Annual International Conference of the IEEE Engineering in Medicine and Biology Society, Vol. 7, 2004, pp. 53445347. 